\title{
MINI-REVIEW
}

\section{Pathomechanisms of Altered Wound Healing in Recessive Dystrophic Epidermolysis Bullosa}

\author{
Francesca Cianfarani, ${ }^{*}$ Giovanna Zambruno, ${ }^{\dagger}$ Daniele Castiglia, ${ }^{*}$ and Teresa Odorisio*
}

From the Laboratory of Molecular and Cell Biology, * Istituto Dermopatico dell'Immacolata-IRCCS, Rome; and the Genetic and Rare Diseases Research Area ${ }^{\dagger}$ Bambino Gesù Children's Hospital-IRCCS, Rome, Italy

\author{
Accepted for publication \\ March 10, 2017. \\ Address correspondence to \\ Teresa Odorisio, Ph.D., \\ Laboratory of Molecular and \\ Cell Biology, Istituto \\ Dermopatico dell'Immacolata- \\ IRCCS, Via dei Monti di \\ Creta, 104, 00167 Roma, \\ Italy. E-mail: t.odorisio@idi.it.
}

\begin{abstract}
Individuals with recessive dystrophic epidermolysis bullosa (RDEB), a rare genetic skin disease, carry mutations in the COL7A1 gene that codes for type VII collagen, an extracellular matrix component of the basement membrane zone forming the anchoring fibrils. As a consequence, RDEB individuals manifest unremitting skin blistering that evolves into chronic wounds, inflammation, and fibrosis. These features play a central role in the development of more severe disease complications, such as mitten deformities of hands and feet and aggressive epithelial cancers. Despite being recognized as a central clinical issue for RDEB, wound healing impairment has been only marginally investigated. Recently, studies with disease mouse models started to shed light on the molecular mechanisms underlying the altered healing response of RDEB. In turn, alterations found in RDEB skin cell behavior fostered the understanding of mechanisms that may be responsible for defective skin repair. This review summarizes findings related to healing impairment in RDEB, and highlights therapeutic strategies for ameliorating healing. (Am J Pathol 2017, 187: 1445-1453; http://dx.doi.org/10.1016/j.ajpath.2017.03.003)
\end{abstract}

Epidermolysis bullosa (EB) is a group of inherited skin diseases characterized by defective epithelial cell adhesion leading to skin fragility and trauma-induced blistering. ${ }^{1}$ Dystrophic EB (DEB), one of the EB major subtypes, is due to mutations in the gene coding for type VII collagen (C7), the main component of anchoring fibrils. These structures guarantee the firm adhesion of the epidermis to the underlying dermis.

Patients affected with the recessive form of DEB (RDEB) may have different disease manifestations, ranging from a few, localized blisters to generalized skin and mucosal blistering. The continuous damage to the epidermal barrier leads to skin infections and persistent inflammation, and healing can be strongly compromised, with chronic wound and scar formation. In turn, the inflammatory and fibrotic skin environment favors the development of further disease complications, including esophageal stenosis, pseudosyndactyly, and aggressive squamous cell carcinomas. ${ }^{2,3}$

In the absence of a disease cure, promoting physiological healing of skin wounds is fundamental to ameliorate life conditions and prolong life expectancy of RDEB individuals. Diverse therapeutic procedures have been attempted. $^{4,5}$ Besides completely or partly restoring C7 expression levels at the dermal-epidermal junction via protein, gene, and cell therapy, new approaches aimed at improving disease morbidities are considered. To develop more effective prohealing therapies, a specific and deeper understanding of the molecular and cellular mechanisms altered in RDEB skin repair is absolutely needed. Recently, a few studies started to shed light on the effects of C7 loss on skin healing, in particular by exploiting animal models of RDEB. Such models are a powerful tool to deepen knowledge on skin alterations and healing defects, thus avoiding invasive studies on affected individuals.

In this review, we highlight the advances in understanding wound healing defects in RDEB.

Supported by Italian Ministry of Health ER-2353637-Eranet (EBThera; D.C.), RC2016-1.2 (D.C.), and RC2016-4.4RIC (T.O.).

Disclosures: None declared. 


\section{Recessive Dystrophic Epidermolysis Bullosa}

EB is a clinically and genetically heterogeneous group of skin blistering diseases due to defects in cell-cell or cellmatrix adhesion components. ${ }^{1}$ Mutations in 1 of 19 different genes have been so far recognized as the cause of EB. ${ }^{1,6,7}$ On the basis of the protein affected and its site of expression, skin cleavage occurs at different levels, within or just beneath the epidermis. DEB is the form in which cleavage occurs at the deepest level, below the cutaneous basement membrane zone in the uppermost dermis (Figure 1A).

DEB can be inherited either as an autosomal dominant or recessive (RDEB) trait, and the recessive form is frequently associated with more severe clinical signs. ${ }^{1}$ Patients experiencing DEB carry mutations in the COL7Al gene encoding for type VII collagen (C7), the main component of anchoring fibrils. ${ }^{8} \mathrm{C} 7$ is a homotrimer consisting of a central triple helical domain flanked by $\mathrm{N}$ - and C-terminal noncollagenous domains. Homotrimers assemble in an antiparallel tail-to-tail manner, linked by disulfide bonds proximal to the $\mathrm{C}$-terminal noncollagenous domain (Figure 1A), and then polymerize in a highly organized manner to form anchoring fibrils. The collagenous portion emanates into the upper dermis and binds covalently to dermal collagen fibrils, whereas the $\mathrm{N}$-terminal domains of anchoring fibril ends interact with laminin-332 and collagen IV at the basement membrane zone ${ }^{8,9}$ Laminin-332, in turn, binds to the $\alpha 6 \beta 4$ integrin receptor of basal keratinocytes. Lack or strongly reduced C7 manifests in anchoring defects of the epidermis and stratified epithelia to the underlying connective tissues, and friction-induced blister formation.

In patients affected with RDEB, repeated blistering leads to protracted wound healing. In particular, at body sites exposed to mechanical stress, continuous cycles of blistering and altered healing lead to persistent ulcerations characterized by incomplete reepithelialization, frequent infections, and perilesional skin inflammation, and, over time, to chronic ulcers, accompanied by fibrosis and scarring (Figures 1B and 2). In the most severe forms, RDEB pathological manifestations include syndactyly, mitten deformities and limb ankylosis, mucosal blistering leading to esophageal strictures, and the development of multiple cutaneous squamous cell carcinomas. ${ }^{1,10}$ Squamous cell carcinomas develop starting from the teenage years, affect $>90 \%$ of patients by the age of 55 years, are characterized by an aggressive biological behavior with a high metastatic rate, and represent the leading cause of death in RDEB patients. ${ }^{10}$

\section{Wound Healing Defects in RDEB}

Skin repair is a complex process during which a highly ordered sequence of events, finely tuned by the molecular crosstalk between different cell populations at the wound site, guarantees efficient recovery of skin homeostasis. ${ }^{11}$
Historically, cutaneous wound healing has been divided into four sequential and partly overlapping phases: hemostasis, inflammation, cell proliferation, and tissue remodeling. Unbalance of one or more of these events may result in two opposite detrimental outcomes: a delay or lack of wound closure and the formation of a hypertrophic scar. ${ }^{12}$ In RDEB patients, diverse factors contribute to impaired healing, leading to alterations in all phases of the repair process, and both chronic wounds and fibrosis are observed (Figures 1, B and C, and 2).

\section{Defects in the Inflammatory Response}

Immediately after injury, blood loss is promptly halted by the formation of a provisional fibrin clot. Platelets trapped within the clot actively release factors that attract inflammatory and other cell types. Neutrophils are the first immune cells reaching the wound bed, followed by monocytes that differentiate into macrophages. Besides removing cell debris and pathogens, these cell populations contribute to generate the milieu rich in cytokines and growth factors necessary for promoting proliferation and migration of the different cell types at the wound site. ${ }^{13}$

Excessive and persistent inflammation is one of the main causes of impaired wound healing. In the skin of patients affected by various forms of EB, the continuous formation of denuded skin lesions frequently leads to bacterial colonization, which may evolve into critical colonization and infection. Wound critical colonization/infection, in turn, activates a burst of inflammation, and may eventually end up into sepsis if not accurately treated. ${ }^{14}$ Colonizing bacteria may form biofilms that are difficult to treat with antimicrobials and interfere with skin cell functions. The molecular mechanisms underlying such interference are still not clarified. Among the pathogens that have been found to massively colonize EB skin lesions, strains of Staphylococcus and Streptococcus were revealed in the vast majority of patients. ${ }^{15}$ Multiple types of Staphylococcus aureus may simultaneously colonize wounds of EB patients, and when this occurs the risk of developing chronic wounds increases compared with colonization by a single type of pathogen. ${ }^{16}$ Flagellated bacterial strains may also contribute to wound inflammation and impaired healing after flagellin-mediated Toll-like receptor 5 activation and up-regulation of the alarmin high mobility group box-1 (HMGB-1). ${ }^{17}$

Accumulating findings indicate an intrinsic proinflammatory state in RDEB skin (Figures $1 \mathrm{C}$ and 2). A transcriptome analysis of RDEB versus normal skin revealed increased expression of several genes involved in immune system activation. ${ }^{18}$ IL receptors IL4R and IL13RA1, the proinflammatory chemokines stromal cell-derived factor 1, CXCL13, monocyte chemoattractant protein-1, and regulated on activation normal $\mathrm{T}$ cell expressed and secreted (RANTES), together with proteins induced by interferon- $\alpha$, were found to be overexpressed in the skin of RDEB individuals. Moreover, defensins, 
A
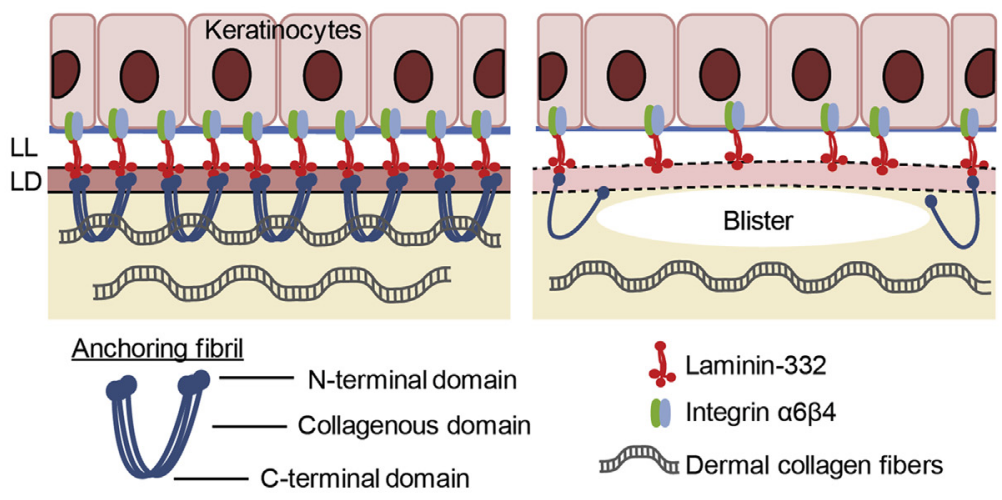

d. Laminin-332

Integrin $\alpha 6 \beta 4$

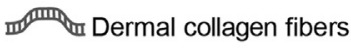
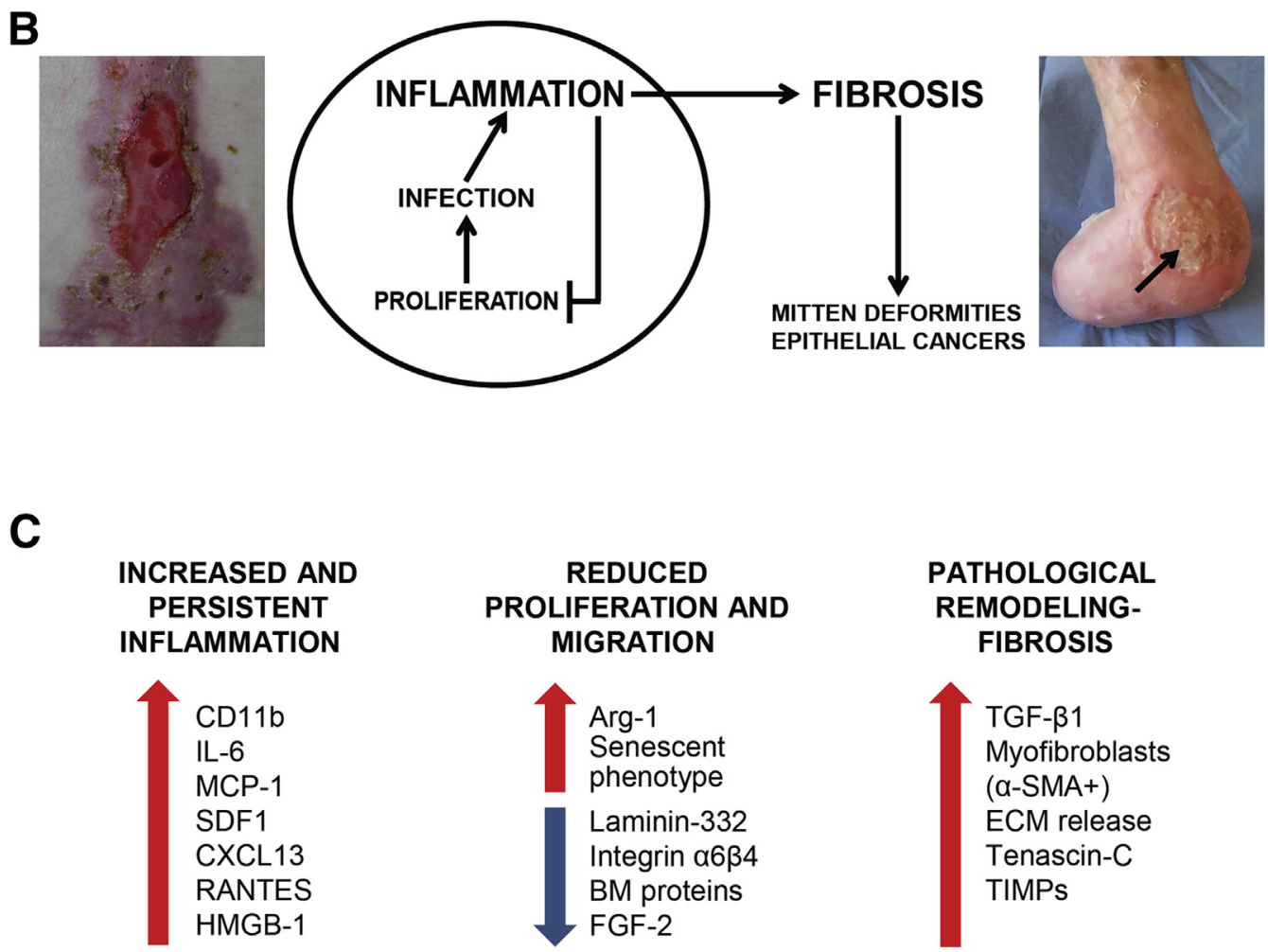

Figure 1 Skin blistering and alterations affecting skin repair in dystrophic epidermolysis bullosa (DEB). A: Schematic representation of anchoring fibril molecular interactions at the dermal-epidermal junction zone, and consequences of their loss or lack in the skin of DEB patients. In normal skin (left panel), type VII collagen (C7) filaments assemble into antiparallel trimers linked in proximity of the C-terminal domain to form anchoring fibrils that are located in the upper dermis. C7 globular N-terminal domains interact with laminin-332 and type IV collagen within the lamina densa (LD) zone of the basement membrane. Laminin-332 trimeric molecules cross the lamina lucida (LL), the upper part of the basement membrane zone, and interact with the a6ß4 integrin complex on keratinocyte cell membrane. In the skin of DEB patients (right panel), C7 mutation(s) variously affect(s) C7 synthesis, secretion, and assembly, resulting into rudimental anchoring fibrils or their absence. As a consequence, the dermal-epidermal junction molecular composition is profoundly altered and the epidermis detaches from the underlying dermis, with blister formation on friction or minor trauma. The cleavage site is located beneath LD. B: The vicious cycle of skin repair in RDEB. The proliferative phase is compromised as a consequence of C7 lack or strong reduction. Wound healing delay favors microbial colonization and infection that, together with repetitive skin wounding, increase and prolong inflammation. Perturbed inflammation further delays skin repair, eventually leading to chronic wound development or scar formation. The fibrotic skin environment favors most severe disease complications: deformities of hands and feet (mitten deformities) and aggressive epithelial cancers. An image of a nonhealing bleeding wound located over a bony prominence surrounded by erythematous inflamed skin is shown in the left panel. The image in the right panel shows mitten foot deformity with digit loss of a patient with severe RDED. A deep chronic wound with raised edges and fibrinous exudate adhering to the wound bed is also visible on the malleolus (arrow). Chronic ulcers may eventually develop into aggressive squamous cell carcinomas. C: Molecular alterations found in RDEB skin/wounded skin and their effects in the different phases of the healing process. $\alpha$-SMA, $\alpha$-smooth muscle actin; FGF, fibroblast growth factor; MCP, monocyte chemoattractant protein; RANTES, regulated on activation normal T cell expressed and secreted; SDF, stromal cell-derived factor; TIMP, tissue inhibitor of metalloproteinase. 
antimicrobial peptides of the innate immune defense system, were also up-regulated, in accordance with the compromised barrier function of RDEB skin.

The comparative analysis of fibroblasts isolated from nonlesional skin of a RDEB monozygotic twin pair with different disease manifestations also showed increased levels of released proinflammatory cytokines IL-6 and monocyte chemoattractant protein-1 by the cells of the twin with the more severe phenotype, in whom blisters and skin lesions affect the entire body surface. ${ }^{19}$ This finding indicates that the inflammatory state of RDEB skin may correlate with disease severity.

Increased levels of the proinflammatory cytokines IL- $1 \beta$, IL-2, IL-6, IL-10, tumor necrosis factor- $\beta$, and interferon- $\gamma$ were found in blood serum of EB patients as compared with healthy controls, and in RDEB patients with respect to other EB subtypes. ${ }^{20}$ Again, IL-6 expression was increased in the more severe EB patients.

A study on the expression of the HMGB-1 proinflammatory mediator in the blood serum of RDEB patients also indicated a correlation with disease severity. ${ }^{21}$ The transcriptional regulator HMGB-1 also functions as an alarmin released by immune cells and necrotic cells that, by binding to membrane receptors (specifically, the receptor for advanced glycation end products and Toll-like receptors 2 and 4), stimulates proinflammatory cytokine secretion. ${ }^{22}$ Nuclear HMGB-1 is expressed in keratinocytes and fibroblasts in nonlesional skin; during wound healing, it is
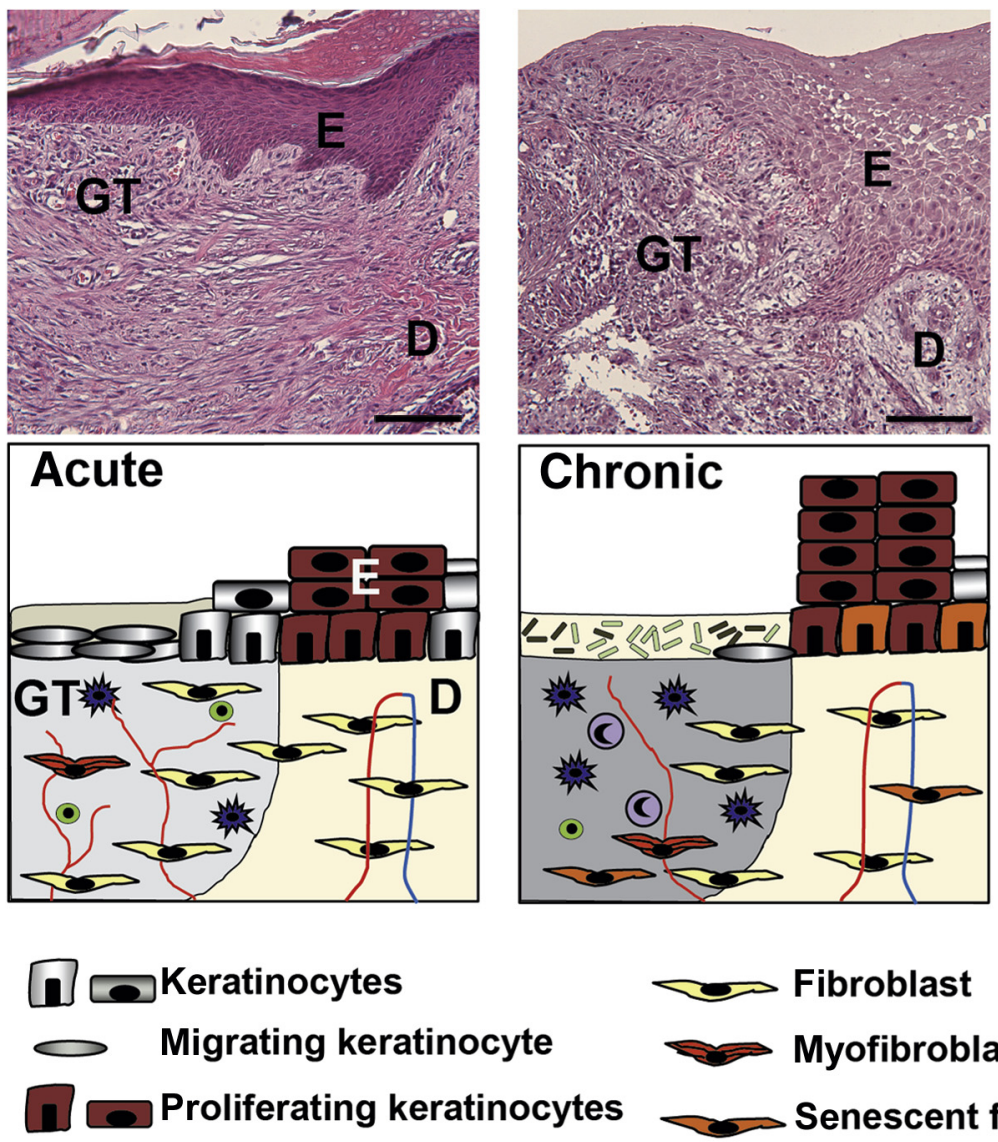

Senescent keratinocyte
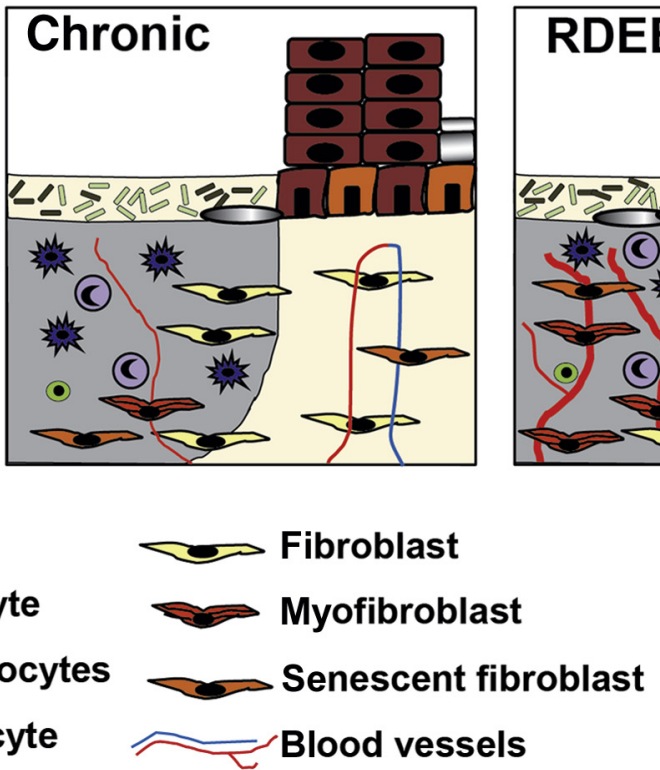
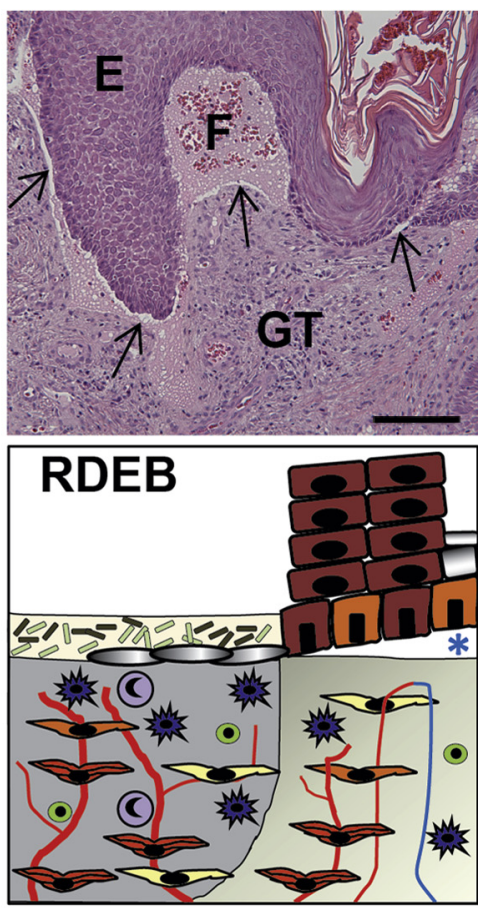

Figure 2 Comparison of main features characterizing acute, chronic, and RDEB wounds. Cartoons depict similarities and differences in wound healing responses in healthy skin (acute healing), chronic ulcers, and RDEB wounds; relative histologies on the top of cartoons represent characteristics of an acute wound in early remodeling phase, a chronic venous leg ulcer margin, and a RDEB leg chronic wound margin. RDEB wounds manifest several features of chronic ulcers: excessive epidermal hyperproliferation (E) at the wound margin and defective keratinocyte migration, frequent bacterial colonization and biofilm formation, excessive and persistent inflammation, perturbed matrix remodeling [indicated by granulation tissue (GT), darker background], and presence of senescent cells. Although in chronic ulcers reepithelialization is almost stuck, RDEB wounds manifest a consistent closure delay, but generally heal. In RDEB wounds located in body sites exposed to trauma, the newly formed epidermis easily detaches from the underlying granulation tissue and adjacent inflamed dermis (asterisk in the cartoon; arrows in the histology) continuously activating the healing responses, as also indicated by the presence of fibrin (F), and favoring chronic wound formation. Angiogenesis is usually reduced in chronic ulcers, whereas it is augmented in RDEB wounds and adjacent dermis. Myofibroblast accumulation further contributes to altered matrix deposition in the granulation tissue of RDEB wounds. Persistent inflammation, excessive ECM deposition due to reduced myofibroblast apoptotic removal, and increased vascularization, all signs of a stiff/profibrotic skin environment, characterize the dermis (D) of RDEB patients (indicated by the darker background). Scale bars $=70 \mu \mathrm{m}$. 
up-regulated and found in the cytoplasmic compartment from where it is released. ${ }^{23}$ HMGB-1 promotes fibroblast and keratinocyte activation, angiogenesis, and scar formation. ${ }^{23,24}$ Elevated HMGB-1 levels have been detected in lesional $\operatorname{skin}^{17}$ and wound fluid ${ }^{25}$ of RDEB patients, where it is mainly released by necrotic keratinocytes.

These findings indicate that inflammatory responses are frequently activated in EB patients, particularly in those affected with RDEB.

The analysis of the $\mathrm{C} 7$ hypomorphic mouse, an animal model of severe RDEB manifesting early blistering, retarded growth, reduced life expectancy, and high incidence of mitten deformities, also indicated the existence of a persistent inflammatory state. ${ }^{26}$ Histochemical characterization of the forepaws, the body location mostly affected by blister formation and evolving in mitten deformities, revealed an increased number of CD11b-positive cells, consisting of granulocytes and monocytes/macrophages. ${ }^{26}$

\section{Defects in the Proliferative Phase}

After the initial inflammatory burst, cells at the wound margin start to actively proliferate to reconstitute the lost tissue. Keratinocytes proliferate and migrate, a process called reepithelialization. Dermal fibroblasts invade the fibrin clot and start to release collagens and other components of the extracellular matrix (ECM). Fibroblasts then differentiate into myofibroblasts, activated cells expressing $\alpha$-smooth muscle actin, and manifesting contractile properties. Once the matrix is released, endothelial cells migrate from the wound margin and assemble into new vessel structures, which promote healing by supplying oxygen and nutrients. This highly cellularized and vascularized tissue, the granulation tissue, which also contains macrophages with an anti-inflammatory phenotype and lymphocytes, transiently fills the wound bed sustaining the metabolically active proliferative phase.

A beneficial effect of a transient burst of senescent cells has been described in normal wound healing. ${ }^{27}$ Senescent fibroblasts and endothelial cells release platelet-derived growth factor-A and vascular endothelial growth factor, thus inducing myofibroblast differentiation and promoting wound closure. However, the persistent presence of senescent cells affects skin repair at chronic wound sites ${ }^{28}$ (Figure 2). Senescent cells manifest a reduced proliferative rate, a reduced lifespan, and the acquisition of a senescenceassociated secretory phenotype, a mixture of inflammatory cytokines, chemokines, growth factors, and proteases that contributes to healing impairment. A transcriptome analysis revealed strong overlapping in gene expression between RDEB skin and skin of elderly individuals. ${ }^{18}$ This senescent cell phenotype is due to the persistent activation of skin cells as a response to continuous blistering and wounding. Moreover, unremitting skin wounds may over time accelerate epidermal stem cell depletion, further affecting reepithelialization. Genes involved in keratinocyte differentiation, particularly filaggrin, and in the formation of the cornified envelope are similarly modulated in RDEB and aged skin. A set of genes up-regulated in RDEB and elderly skin code for collagens, specifically, collagen I, IV, and VI, accounting for the thickened, profibrotic appearance of RDEB skin.

Another transcriptome analysis performed by comparing gene expression between chronic wound margin and unwounded RDEB skin revealed increased arginase-1 (ARGl) gene expression, as well as alterations of other genes involved in L-arginine metabolism. ${ }^{29} \mathrm{~L}$-arginine is the substrate for nitric oxide synthesis, catalyzed by nitric oxide synthases. On skin injury, inducible nitric oxide synthase-mediated nitric oxide up-regulation stimulates diverse biological events, including reepithelialization, cell migration, collagen synthesis, and wound contraction. ${ }^{30}$ Thus, altered L-arginine metabolism may contribute to the healing defect of RDEB skin by impairing the proliferative phase.

An important insight into the molecular mechanisms underlying alterations in the proliferative phase of RDEB wounds comes from the analysis of the $\mathrm{C} 7$ hypomorphic mice and the tamoxifen-inducible Col7al knockout mice, which manifest lack of $\mathrm{C} 7$ after topical tamoxifen treatment, but not chronic disease manifestations. ${ }^{31}$ This analysis revealed that $\mathrm{C} 7$ lack delays wound healing by exerting a dual effect. On the epidermal side, it perturbs laminin-332 deposition and integrin $\alpha 6 \beta 4$ distribution at the basal membrane zone. As a consequence, activation of laminin332/integrin a6ß34 signaling pathways, specifically c-Jun $\mathrm{N}$-terminal kinase (JNK)2 and AKT, is altered and keratinocyte migration affected. More important, perturbations in laminin-332, integrin $\alpha 6 \beta 4$, and their signaling pathways were also observed in acute and chronic wounds of RDEB patients. ${ }^{31}$ On the dermal side, lack of C7 impairs fibroblast migration and granulation tissue maturation. This effect associates with increased transforming growth factor (TGF)$\beta 1$ expression and signaling (SMAD2 activation), and collagen I deposition, signs of a fibrotic microenvironment, whereas fibroblast growth factor-2 expression is decreased. Recombinant $\mathrm{C} 7$ addition to cultured mouse keratinocytes and fibroblasts lacking Col7al is able to revert altered cell behavior, indicating that the observed defects are a direct consequence of $\mathrm{C} 7$ lack. Consistent with these findings, Wang et $\mathrm{al}^{32}$ showed that recombinant $\mathrm{C} 7$ topical application in athymic nude mice accelerates wound closure by promoting reepithelialization.

\section{Defects in Skin Remodeling}

Remodeling is a long-lasting phase characterized by the reconstitution of the epidermal barrier, apoptotic cell loss, and ECM reorganization. Programmed cell death leads to vessel regression, inflammatory cell reduction, and myofibroblast disappearance. Persistent inflammation, reduced myofibroblast removal, and excessive ECM deposition are 
all signs of fibrosis due to a defective remodeling phase (Figure 2).

Skin fibrosis is a central burden for RDEB, as the fibrotic microenvironment is regarded as the leading cause for the development of hand and foot deformities and of aggressive squamous cell carcinomas ${ }^{2,33}$ (Figure 1B). A central role in the onset of a fibrotic environment and scar formation is played by the TGF- $\beta$, mainly by the isoform 1 (TGF- $\beta 1$ ), the most abundantly expressed during wound healing. TGF- $\beta 1$ is released by different cell types at the wound site and involved in all stages of wound healing. ${ }^{34}$ When overexpressed, TGF- $\beta 1$ promotes fibrosis by stimulating fibroblast differentiation into myofibroblasts, which are responsible for wound contraction and abundant release of ECM proteins. TGF- $\beta$ acts by binding to the TGF- $\beta$ R complex, thus initiating an intracellular-specific signaling cascade involving the SMAD proteins, as well as other nonspecific signaling pathways. ${ }^{35}$

An analysis of gene expression performed in scars obtained from RDEB patients compared to non-RDEB scars, to RDEB skin and skin from healthy donors identified significantly increased IL-1 $\beta$, IL- 6 , and tenascin-C levels in RDEB scars, indicative of a persistent proinflammatory state. $^{2}$ Tenascin-C is an ECM glycoprotein transiently expressed during wound healing. Its overexpression in the skin was shown to induce persistent fibrosis through binding to the Toll-like receptor 4, a receptor mediating innate immune response through release of proinflammatory cytokines. ${ }^{36}$

Proteome analysis of secreted ECM and conditioned medium of dermal fibroblasts isolated from severe RDEB individuals revealed profound alterations when compared with healthy donors. ${ }^{37}$ Although not assessed in a wound healing context, this analysis indicates that lack of $\mathrm{C7}$ profoundly affects fibroblast activity with a negative impact on their function during skin repair. Again, most relevant alterations found in proteins secreted by RDEB fibroblasts regard increased interstitial collagen and tenascin-C deposition, and TGF- $\beta$ release; a reduced amount of basement membrane proteins (laminin $\beta 1$, laminin $\gamma 1$, collagen IV, nidogen 1 , and fibulin 1) was also shown in this study. An important aspect for ECM remodeling concerns the activity of matrix metalloproteinases, which selectively digest ECM components. Although RDEB fibroblasts manifest reduced proteolytic activity, these cells have been found to secrete increased amounts of some matrix metalloproteinases. ${ }^{37}$ This apparent discrepancy can be explained by the concomitantly increased levels of matrix metalloproteinase inhibitors (tissue inhibitors of metalloproteinases), such as tissue inhibitor of metalloproteinase 3. mRNA expression profiling of fibroblasts isolated from RDEB individuals also indicated that these cells manifest several molecular alterations as compared with normal fibroblasts, and are similar to activated cancer-associated fibroblasts. ${ }^{38} \mathrm{C} 7$ reduction led to increased expression of ECM components and adhesion proteins, such as type $\mathrm{V}$ and XII collagens, the TGF- $\beta$ activator thrombospondin-1, Wnt-5A, and integrin $\alpha 3$ and $\alpha 6$ subunits. These molecular features contribute to a permissive environment for tumor development.

Enhanced TGF- $\beta$ and $\alpha$-smooth muscle actin expression were also found in skin fibroblasts isolated from a RDEB patient as compared to those of his monozygotic twin manifesting a milder phenotype. ${ }^{19}$ TGF- $\beta$ signaling pathways involving SMADs and mitogen-activated protein kinases were also more activated in this sibling. As monozygotic twins are virtually identical, such differences highlight a possible role for TGF- $\beta$ in modifying disease severity.

Phenotypic characterization of the $\mathrm{C} 7$ hypomorphic mouse further revealed a high number of $\alpha$-smooth muscle actin-positive myofibroblasts associated with accumulation of TGF- $\beta$ and tenascin-C, indicative of a fibrotic skin environment. $^{26}$

C7 administration to skin wounds of hypomorphic and athymic wounded mice was associated with decreased fibrosis. ${ }^{26,32}$ In the wounds of athymic mice, myofibroblast number, collagen deposition, and expression of connective tissue growth factor, which mediates TGF- $\beta$ activity in inducing fibroblast differentiation into myofibroblasts, were reduced, and the relative amount of the different TGF- $\beta$ isoforms was changed, with the profibrotic TGF- $\beta 2$ isoform being less expressed and the antifibrotic TGF- $\beta 3$ being more expressed.

\section{Promoting Healing}

In the absence of effective therapeutic treatments, the first line of intervention for promoting quality of life for RDEB patients is wound care. Debridement, control of wound infection by topical antiseptics and antibiotics, and the use of proper dressings promote healing and/or reduce pain. ${ }^{39}$ In general, dressings should absorb exudate, generate a moist environment, provide mechanical protection, and be nonadherent to reduce trauma and pain at removal. ${ }^{40} \mathrm{An}$ international consensus approach to EB wound care lists recommendations that take into consideration EB type, patient age and general health condition, pain and itching management, caregiver education, and specific wound characteristics (location, size, need for debridement, inflammation, critical colonization/infection, wound bed, and edge appearance, etc). ${ }^{41}$ Critical aspects for wound healing in RDEB patients comprise malnutrition due to mucosal blistering as well as chronic multifactorial anemia, hypoalbuminemia, and persistently elevated acute phase reactants mainly consequent to chronic and extensive skin denudation. Optimization of nutrition and effective treatment of these complications are essential, although rarely achievable goals, for effective wound management in RDEB patients.

A therapeutic option for the treatment of chronic wounds in RDEB patients is the application of autologous or allogeneic skin grafts. ${ }^{42}$ Decellularized amniotic grafts have 
also been used to improve healing of RDEB patients. ${ }^{43}$ Cellular and acellular matrices exert a prohealing effect by reconstituting tissue continuity and by supporting cellular activity, providing growth factors and cytokines. Topical treatment with compounds promoting healing functions has also been tested. On the basis of the observation of a significant improvement in wound healing after granulocyte colony-stimulating factor treatment before and after bone marrow transplantation of a RDEB patient, a pilot trial has been performed in a limited number of patients to evaluate the effect and safety of granulocyte colony-stimulating factor topical administration on wound healing. ${ }^{44}$ Granulocyte colony-stimulating factor acts by promoting neutrophil mobilization, proliferation, and differentiation. Treatment was safe, and six of seven DEB patients showed reduction in lesion size and blister/erosion count. However, the lifelong use on large body areas of these advanced treatment modalities, as required for RDEB patient management, is limited by practical considerations.

Because RDEB depends on the defective production of $\mathrm{C} 7$, that not only determines blister formation, but also directly affects wound healing, ${ }^{31}$ restoring $\mathrm{C} 7$ expression levels in the RDEB context is the straightforward option to promote skin repair. Gene therapy, protein therapy, and cell therapy approaches aimed at expressing $\mathrm{C} 7$ at the dermalepidermal junction have been tested at preclinical and clinical levels. ${ }^{4,5,45}$ Although some success was obtained with these therapeutic strategies, efficacy and safety still remain main issues for their applicability.

As for gene therapy, viral vector-based approaches have been applied to restore $\mathrm{C} 7$ expression in autologous skin cells and skin equivalents at the preclinical level, ${ }^{46}$ and are now being tested in clinical trials (http://clinicaltrials.gov; trial identifiers NCT01263379, NCT02984085, NCT02493816, and NCT02810951). Recently, a clinical trial with retroviral COL7Al-corrected autologous epidermal grafts transplanted onto wounds of RDEB patients revealed healing improvement in the short-term. ${ }^{47}$ Such effect, however, declined in the long-term. To overcome safety issues linked to insertional mutagenesis and viral-vector potential infection, new enzyme-based methods aimed at editing COL7Al gene defects represent a promising therapeutic tool. Platforms for gene editing use zinc-finger nucleases, transcription activator-like effector nucleases, or clustered regularly interspaced short palindromic repeats-Cas9 nuclease. Some in vitro studies report the feasibility of such approaches for correcting COL7Al gene defects. ${ }^{45,48}$

Skin lesions involve a large part of the body surface in RDEB patients, and blistering also affects the eye, oral, esophageal, and genitourinary mucosae. To obtain a more effective therapy, systemic stem cell or recombinant C7 administration is preferable with respect to local skin treatments. In preclinical studies, intravenously injected recombinant $\mathrm{C} 7$ has been found to specifically localize at sites of skin lesions, where it incorporates into the basement membrane zone, forms anchoring fibrils, and improves wound healing. ${ }^{49}$ Allogeneic blood and bone marrow were also transplanted after myeloablative chemotherapy to RDEB affected children. Hematopoietic and nonhematopoietic stem cells likely homed to sites of injury with increased C7 expression, long-lasting improvement in wound healing, and decreased mucocutaneous blistering in most patients. ${ }^{50}$ The enthusiasm on these results was tempered by the high mortality rate recorded. Allogeneic mesenchymal stem cell intravenous administration has also been tried. Despite no detectable increase in C7 or in anchoring fibrils, improvement was observed in wound healing. ${ }^{51}$

Finally, the technology of induced pluripotent stem cells has been applied to RDEB cells in preclinical studies. Induced pluripotent stem cells were obtained by genetically reprogramming keratinocytes or fibroblasts isolated from RDEB individuals, and COL7Al-corrected induced pluripotent stem cells differentiated into keratinocytes were shown to be able to in vitro reconstitute a stratified epidermis and secrete $\mathrm{C} 7$ at the basement membrane zone. ${ }^{52}$ Moreover, gene-corrected induced pluripotent stem cells obtained from fibroblasts of the $\mathrm{C} 7$ hypomorphic mice were redifferentiated into fibroblasts and administered intradermally to the same RDEB mouse model. Gene-corrected cells were able to increase $\mathrm{C} 7$ at the basement membrane and to improve skin resistance. ${ }^{53}$ These proof-of-principle data suggest that induced pluripotent stem cell-based cell therapy may be considered for RDEB.

Therapeutic approaches aimed at counteracting and lessening or delaying disease manifestations, in particular the inhibition of persistent skin inflammation and resulting fibrosis, are also regarded as an alternative to improve disease course and quality of life of RDEB patients. On the basis of the increasing body of experimental findings indicating TGF- $\beta$ as a major player in the proinflammatory/ profibrotic RDEB skin environment, ${ }^{19,26,37}$ compounds counteracting TGF- $\beta$ activity are being considered as symptom-relief treatments. Recently, the angiotensin inhibitor losartan, which has been shown to lessen TGF- $\beta$ activity and fibrosis, has been used to systemically treat $\mathrm{C} 7$ hypomorphic mice. ${ }^{54}$ The skin of treated mice manifests decreased signs of excessive fibrosis, in particular reduced collagen deposition, collagen fibril thickening, elastic fiber disarrangement, and fibroblast conversion to myofibroblasts. These effects are associated with inhibition of TGF- $\beta$ signaling, and reduced expression of tenascin-C, fibronectin, $\alpha$-smooth muscle actin, and the inflammatory markers galectin-3, IL-6, and tumor necrosis factor- $\alpha$. Consequences of excessive fibrosis, such as pseudosyndactyly and digit loss, were also limited after losartan treatment. As expected, the formation of skin lesions was not prevented in losartantreated mice. However, the reported effects of losartan on healing promotion in other tissues ${ }^{55}$ and on skin repair improvement ${ }^{56}$ indicate that this treatment has the potential to also positively affect wound healing in RDEB. On the 
basis of these encouraging preclinical data, a clinical trial of losartan in RDEB is foreseen.

\section{Conclusions}

Impaired wound healing is a major health problem for patients affected with RDEB. Several lines of evidence indicate that chronic wounds and scar formation are the link between continuous blistering and disease complications. The analysis of RDEB mouse models clearly indicates that lack of C7 not only determines skin fragility, but also directly affects wound healing by impairing skin cell activity. Transcriptome and proteome analyses also revealed that C7 loss profoundly affects RDEB skin cell behavior in both the epidermal and dermal compartments, indicating that the epithelial-mesenchymal molecular crosstalk sustaining healing in all its phases is likely altered. Recovering C7 levels at the epidermis-dermis boundary is, therefore, the primary advanced therapeutic option to restore skin repair. Several findings indicate a direct correlation between skin inflammation and disease severity in RDEB individuals. Excessive and persistent inflammation is a result of repeated skin injury and delayed healing, contributing to both chronic wound development and fibrosis. Thus, targeting inflammatory mediators could be an alternative therapeutic option aimed at mitigating disease manifestations. Further studies specifically investigating molecular and cellular defects in RDEB skin repair are needed for improving the highly disabling and life-threatening manifestations of this incurable disease.

\section{References}

1. Fine JD, Bruckner-Tuderman L, Eady RA, Bauer EA, Bauer JW, Has C, Heagerty A, Hintner H, Hovnanian A, Jonkman MF, Leigh I, Marinkovich MP, Martinez AE, McGrath JA, Mellerio JE, Moss C, Murrell DF, Shimizu H, Uitto J, Woodley D, Zambruno G: Inherited epidermolysis bullosa: updated recommendations on diagnosis and classification. J Am Acad Dermatol 2014, 70:1103-1126

2. Breitenbach J, Gruber C, Klausegger A, Trost A, Bogner B, Reitsamer H, Bauer JW: Pseudosyndactyly: an inflammatory and fibrotic wound healing disorder in recessive dystrophic epidermolysis bullosa. J Dtsch Dermatol Ges 2015, 13:1257-1266

3. Mittapalli R, Madl J, Löffek S, Kiritsi D, Kern JS, Römer W, Nyström A, Bruckner-Tuderman L: Injury-driven stiffening of the dermis expedites skin carcinoma progression. Cancer Res 2016, 76: 940-951

4. Has C, Kiritsi D: Therapies for inherited skin fragility disorders. Exp Dermatol 2015, 24:325-331

5. Woodley DT, Chen M: Recessive dystrophic epidermolysis bullosa: advances in the laboratory leading to new therapies. J Invest Dermatol 2015, 135:1705-1707

6. Lin Z, Li S, Feng C, Yang S, Wang H, Ma D, Zhang J, Gou M, Bu D, Zhang T, Kong X, Wang X, Sarig O, Ren Y, Dai L, Liu H, Zhang J, Li F, Hu Y, Padalon-Brauch G, Vodo D, Zhou F, Chen T, Deng H, Sprecher E, Yang Y, Tan X: Stabilizing mutations of KLHL24 ubiquitin ligase cause loss of keratin 14 and human skin fragility. Nat Genet 2016, 48:1508-1516

7. He Y, Maier K, Leppert J, Hausser I, Schwieger-Briel A, Weibel L, Theiler M, Kiritsi D, Busch H, Boerries M, Hannula-Jouppi K,
Heikkilä H, Tasanen K, Castiglia D, Zambruno G, Has C: Monoallelic mutations in the translation initiation codon of KLHL24 cause skin fragility. Am J Hum Genet 2016, 99:1395-1404

8. Chung HJ, Uitto J: Type VII collagen: the anchoring fibril protein at fault in dystrophic epidermolysis bullosa. Dermatol Clin 2010, 28 93-105

9. Brittingham R, Uitto J, Fertala A: High-affinity binding of the NC-1 domain of collagen VII to laminin 5 and collagen IV. Biochem Biophys Res Commun 2006, 343:692-699

10. Fine JD, Johnson LB, Weiner M, Li KP, Suchindran C: Epidermolysis bullosa and the risk of life-threatening cancers: the National EB Registry Experience, 1986-2006. J Am Acad Dermatol 2009, 60:203-211

11. Gurtner GC, Werner S, Barrandon Y, Longaker MT: Wound repair and regeneration. Nature 2008, 453:314-321

12. Eming SA, Martin P, Tomic-Canic M: Wound repair and regeneration: mechanisms, signaling, and translation. Sci Transl Med 2014, 6: $265 \mathrm{sr} 6$

13. Eming SA, Hammerschmidt M, Krieg T, Roers A: Interrelation of immunity and tissue repair or regeneration. Semin Cell Dev Biol 2009, 20:517-527

14. van der Kooi-Pol MM, Duipmans JC, Jonkman MF, van Dijl JM: Host-pathogen interactions in epidermolysis bullosa patients colonized with Staphylococcus aureus. Int J Med Microbiol 2014, 304: 195-203

15. Brandling-Bennett HA, Morel KD: Common wound colonizers in patients with epidermolysis bullosa. Pediatr Dermatol 2010, 27:25-28

16. van der Kooi-Pol MM, de Vogel CP, Westerhout-Pluister GN, Veenstra-Kyuchukova YK, Duipmans JC, Glasner C, Buist G, Elsinga GS, Westra H, Bonarius HP, Groen H, van Wamel WJ, Grundmann H, Jonkman MF, van Dijl JM: High anti-staphylococcal antibody titers in patients with epidermolysis bullosa relate to longterm colonization with alternating types of Staphylococcus aureus. J Invest Dermatol 2013, 133:847-850

17. Hoste E, Arwert EN, Lal R, South AP, Salas-Alanis JC, Murrell DF, Donati G, Watt FM: Innate sensing of microbial products promotes wound-induced skin cancer. Nat Commun 2015, 6:5932

18. Breitenbach JS, Rinnerthaler M, Trost A, Weber M, Klausegger A, Gruber C, Bruckner D, Reitsamer HA, Bauer JW, Breitenbach M: Transcriptome and ultrastructural changes in dystrophic epidermolysis bullosa resemble skin aging. Aging 2015, 7:389-411

19. Odorisio T, Di Salvio M, Orecchia A, Di Zenzo G, Piccinni E, Cianfarani F, Travaglione A, Uva P, Bellei B, Conti A, Zambruno G, Castiglia D: Monozygotic twins discordant for recessive dystrophic epidermolysis bullosa phenotype highlight the role of TGF- $\beta$ signalling in modifying disease severity. Hum Mol Genet 2014, 23: $3907-3922$

20. Esposito S, Guez S, Orenti A, Tadini G, Scuvera G, Corti L, Scala A, Biganzoli E, Berti E, Principi N: Autoimmunity and cytokine imbalance in inherited epidermolysis bullosa. Int J Mol Sci 2016, 17:1625

21. Petrof G, Abdul-Wahab A, Proudfoot L, Pramanik R, Mellerio JE, McGrath JA: Serum levels of high mobility group box 1 correlate with disease severity in recessive dystrophic epidermolysis bullosa. Exp Dermatol 2013, 22:417-437

22. Bianchi ME: HMGB1 loves company. J Leukoc Biol 2009, 89: $573-576$

23. Dardenne AD, Wulff BC, Wilgus TA: The alarmin HMGB-1 influences healing outcomes in fetal skin wounds. Wound Repair Regen 2013, 21:282-291

24. Pandolfi F, Altamura S, Frosali S, Conti P: Key role of DAMP in inflammation, cancer, and tissue repair. Clin Ther 2016, 38:1017-1028

25. Tamai K, Yamazaki T, Chino T, Ishii M, Otsuru S, Kikuchi Y, Iinuma S, Saga K, Nimura K, Shimbo T, Umegaki N, Katayama I, Miyazaki J, Takeda J, McGrath JA, Uitto J, Kaneda Y: PDGFRalphapositive cells in bone marrow are mobilized by high mobility group box 1 (HMGB1) to regenerate injured epithelia. Proc Natl Acad Sci U S A 2011, 108:6609-6614 
26. Fritsch A, Loeckermann S, Kern JS, Braun A, Bösl MR, Bley TA, Schumann H, von Elverfeldt D, Paul D, Erlacher M, Berens von Rautenfeld D, Hausser I, Fässler R, Bruckner-Tuderman L: A hypomorphic mouse model of dystrophic epidermolysis bullosa reveals mechanisms of disease and response to fibroblast therapy. J Clin Invest 2008, 118:1669-1679

27. Demaria M, Ohtani N, Youssef SA, Rodier F, Toussaint W, Mitchell JR, Laberge RM, Vijg J, Van Steeg H, Dollé ME, Hoeijmakers JHJ, de Bruin A, Hara E, Campisi J: An essential role for senescent cells in optimal wound healing through secretion of PDGF-AA. Dev Cell 2014, 31:722-733

28. Wall IB, Moseley R, Baird DM, Kipling D, Giles P, Laffafian I, Price PE, Thomas DW, Stephens P: Fibroblast dysfunction is a key factor in the non-healing of chronic venous leg ulcers. J Invest Dermatol 2008, 128:2526-2540

29. Wessagowit V, Mallipeddi R, McGrath JA, South AP: Altered expression of L-arginine metabolism pathway genes in chronic wounds in recessive dystrophic epidermolysis bullosa. Clin Exp Dermatol 2004, 29:664-668

30. Isenberg JS, Ridnour LA, Grahamespey M, Wink DA, Roberts DD: Nitric oxide in wound-healing. Microsurgery 2005, 25:442-451

31. Nyström A, Velati D, Mittapalli VR, Fritsch A, Kern JS, BrucknerTuderman L: Collagen VII plays a dual role in wound healing. J Clin Invest 2013, 123:3498-3509

32. Wang X, Ghasri P, Amir M, Hwang B, Hou Y, Khilili M, Lin A, Keene D, Uitto J, Woodley DT, Chen M: Topical application of recombinant type VII collagen incorporates into the dermal-epidermal junction and promotes wound closure. Mol Ther 2013, 21: $1335-1344$

33. Lim YL, South AP: Tumour-stroma crosstalk in the development of squamous cell carcinoma. Int J Biochem Cell Biol 2014, 53:450-458

34. Barrientos S, Stojadinovic O, Golinko MS, Brem H, Tomic-Canic M: Growth factors and cytokines in wound healing. Wound Repair Regen 2008, 16:585-601

35. Massagué J, Gomis RR: The logic of TGFbeta signaling. FEBS Lett 2006, 580:2811-2820

36. Bhattacharyya S, Wang W, Morales-Nebreda L, Feng G, Wu M, Zhou X, Lafyatis R, Lee J, Hinchcliff M, Feghali-Bostwick C, Lakota K, Budinger GRS, Raparia K, Tamaki Z, Varga J: Tenascin-C drives persistence of organ fibrosis. Nat Commun 2016, 7:11703

37. Küttner V, Mack C, Rigbolt KT, Kern JS, Schilling O, Busch H, Bruckner-Tuderman L, Dengjel J: Global remodelling of cellular microenvironment due to loss of collagen VII. Mol Syst Biol 2013, 9:657

38. Ng Y-Z, Pourreyron C, Salas-Alanis JC, Dayal JHS, CepedaValdes R, Yan W, Wright S, Chen M, Fine JD, Hogg FJ, McGrath JA, Murrell DF, Leigh IM, Lane EB, South AP: Fibroblastderived dermal matrix drives development of aggressive cutaneous squamous cell carcinoma in patients with recessive dystrophic epidermolysis bullosa. Cancer Res 2012, 72:3522-3534

39. Denyer JE: Wound management for children with epidermolysis bullosa. Dermatol Clin 2010, 28:257-264

40. Grocott P, Blackwell R, Weir H, Pillay E: Living in dressings and bandages: findings from workshops with people with epidermolysis bullosa. Int Wound J 2013, 10:274-284

41. Pope E, Lara-Corrales I, Mellerio J, Martinez A, Schultz G, Burrell R, Goodman L, Coutts P, Wagner J, Allen U, Sibbald G: A consensus approach to wound care in epidermolysis bullosa. J Am Acad Dermatol 2012, 67:904-917

42. Natsuga K, Sawamura D, Goto M, Homma E, Goto-Ohguchi Y, Aoyagi S, Akiyama M, Kuroyanagi Y, Shimizu H: Response of intractable skin ulcers in recessive dystrophic epidermolysis bullosa patients to an allogeneic cultured dermal substitute. Acta Derm Venereol 2010, 90:165-169

43. Lo V, Lara-Corrales I, Stuparich A, Pope E: Amniotic membrane grafting in patients with epidermolysis bullosa with chronic wounds. J Am Acad Dermatol 2010, 62:1038-1044

44. Fine JD, Manes B, Frangoul H: Systemic granulocyte colonystimulating factor (G-CSF) enhances wound healing in dystrophic epidermolysis bullosa (DEB): results of a pilot trial. J Am Acad Dermatol 2015, 73:56-61

45. Perdoni C, Osborn MJ, Tolar J: Gene editing toward the use of autologous therapies in recessive dystrophic epidermolysis bullosa. Transl Res 2016, 168:50-58

46. Titeux M, Pendaries V, Hovnanian A: Gene therapy for recessive dystrophic epidermolysis bullosa. Dermatol Clin 2010, 28: 361-366

47. Siprashvili Z, Nguyen NT, Gorell ES, Loutit K, Khuu P, Furukawa LK, Lorenz HP, Leung TH, Keene DR, Rieger KE, Khavari P, Lane AT, Tang JY, Marinkovich MP: Safety and wound outcomes following genetically corrected autologous epidermal grafts in patients with recessive dystrophic epidermolysis bullosa. JAMA 2016, 316:1808-1817

48. Osborn MJ, Starker CG, McElroy AN, Webber BR, Riddle MJ, Xia L, DeFeo AP, Gabriel Schmidt RM, von Kalle C, Carlson DF, Maeder ML, Joung JK, Wagner JR, Voytas DF, Blazar BR, Tolar J: TALEN-based gene correction for epidermolysis bullosa. Mol Ther 2013, 21:1151-1159

49. Woodley DT, Wang X, Amir M, Hwang B, Remington J, Hou Y, Uitto J, Keene D, Chen M: Intravenously injected recombinant human type VII collagen homes to skin wounds and restores skin integrity of dystrophic epidermolysis bullosa. J Invest Dermatol 2013, 133:1910-1913

50. Tolar J, Wagner JE: Allogeneic blood and bone marrow cells for the treatment of severe epidermolysis bullosa: repair of the extracellular matrix. Lancet 2013, 382:1214-1223

51. Petrof G, Lwin SM, Martinez-Queipo M, Abdul-Wahab A, Tso S, Mellerio JE, Slaper-Cortenbach I, Boelens JJ, Tolar J, Veys P, Ofuya M, Peacock JL, Martinez AE, McGrath JA: Potential of systemic allogeneic mesenchymal stromal cell therapy for children with recessive dystrophic epidermolysis bullosa. J Invest Dermatol 2015, $135: 2319-2321$

52. Sebastiano V, Zhen HH, Haddad B, Bashkirova E, Melo SP, Wang P, Leung TL, Siprashvili Z, Tichy A, Li J, Ameen M, Hawkins J, Lee S, Li L, Schwertschkow A, Bauer G, Lisowski L, Kay MA, Kim SK, Lane AT, Wernig M, Oro AE: Human COL7A1-corrected induced pluripotent stem cells for the treatment of recessive dystrophic epidermolysis bullosa. Sci Transl Med 2014, 6:264ra163

53. Wenzel D, Bayerl J, Nyström A, Bruckner-Tuderman L, Meixner A, Penninger JM: Genetically corrected iPSCs as cell therapy for recessive dystrophic epidermolysis bullosa. Sci Transl Med 2014, 6: 264ra165

54. Nyström A, Thriene K, Mittapalli V, Kern JS, Kiritsi D, Dengjel J, Bruckner-Tuderman L: Losartan ameliorates dystrophic epidermolysis bullosa and uncovers new disease mechanisms. EMBO Mol Med 2015, 7:1211-1228

55. Gharaibeh B, Chun-Lansinger Y, Hagen T, McNeill Ingham SJ, Wright V, Fu F, Huard J: Biological approaches to improve skeletal muscle healing after injury and disease. Birth Defects Res C Embryo Today 2012, 96:82-94

56. Kamber M, Papalazarou V, Rouni G, Papageorgopoulou E, Papalois A, Kostourou V: Angiotensin II inhibitor facilitates epidermal wound regeneration in diabetic mice. Front Physiol 2015, $6: 1-8$ 